Quim. Nova, Vol. 36, No. 9, 1296-1302, 2013

\title{
DETERMINAÇÃO DE NIMESULIDA POR ANÁLISE POR INJEÇÃO EM FLUXO COM DETECÇÃO AMPEROMÉTRICA DE MÚLTIPLOS PULSOS
}

\author{
Amanda Barbosa Lima, Sandro Cruz Chaves e Leonardo Morais da Silva \\ Departamento de Química - Universidade Federal dos Vales do Jequitinhonha e Mucuri, MG, Brasil \\ Polyana Fernandes Pereira e Eduardo Mathias Richter \\ Instituto de Química - Universidade Federal de Uberlândia, MG, Brasil \\ Wallans Torres Pio dos Santos* \\ Departamento de Farmácia - Universidade Federal dos Vales do Jequitinhonha e Mucuri, MG, Brasil
}

Recebido em 25/4/13; aceito em 17/5/13; publicado na web em 1/7/13

\begin{abstract}
DETERMINATION OF NIMESULIDE BY FLOW INJECTION ANALYSIS WITH MULTIPLE-PULSE AMPEROMETRIC DETECTION. A simple and fast method for the determination of nimesulide (NI) using flow injection analysis with multiple-pulse amperometric (FIA-MPA) detection at a boron-doped diamond (BDD) electrode was developed. The method was based mainly on the application of a four-potential waveform, $\mathrm{E}_{1(\mathrm{det})}=-0.8 \mathrm{~V} / 30 \mathrm{~ms}, \mathrm{E}_{2(\mathrm{det})}=0.6 \mathrm{~V} / 30 \mathrm{~ms}, \mathrm{E}_{3(\mathrm{det})}=-0.4 \mathrm{~V} / 30 \mathrm{~ms}$ and $\mathrm{E}_{4(\text { cleaning) }}=-0.45 \mathrm{~V} /$ $300 \mathrm{~ms}$ versus $\mathrm{Ag} / \mathrm{AgCl}\left(3.0 \mathrm{~mol} \mathrm{~L}^{-1} \mathrm{KCl}\right)$. NI was detected at three different electrode potentials, at which the nitro group undergoes different redox reactions. The proposed method was selective and sensitive (detection limit of $81.0 \mathrm{nmol} \mathrm{L}^{-1}$ ), and successfully applied for the determination of NI in pharmaceutical formulations, yielding similar results to those obtained by the reference method.
\end{abstract}

Keywords: pulse amperometric detection; nimesulide; boron-doped diamond.

\section{INTRODUÇÃO}

A Nimesulida (NI), $N$-(4-nitro-2-fenoxifenil)metanossulfonamida, é um anti-inflamatório não-esteroide de alta atividade analgésica e antipirética, sendo amplamente prescrita e recomendada por apresentar melhor eficácia sobre os medicamentos similares, como ibuprofeno, diclofenaco e piroxicam. ${ }^{1}$ Além disso, a NI possui menor acidez $(\mathrm{pKa}=6,9) \mathrm{e}$, consequentemente, melhor tolerabilidade gástrica quando comparada aos fármacos utilizados para a mesma finalidade, como o ácido acetilsalicílico $(\mathrm{pKa}=3,4) .{ }^{2}$ Devido às vantagens apresentadas pela NI, este composto está entre os dez medicamentos mais vendidos no Brasil nos últimos anos. ${ }^{3}$ Neste contexto, este analito pode também ser classificado como um poluente orgânico emergente em amostras ambientais, como seus similares supracitados. ${ }^{4}$ Dessa forma, torna-se fundamental o desenvolvimento de métodos analíticos rápidos, simples e de baixo custo que possam ser utilizados no controle de qualidade deste fármaco em formulações farmacêuticas, bem como sua quantificação sensível e seletiva em amostras ambientais.

A Farmacopeia Brasileira indica o doseamento da NI por dois métodos: espectrofotometria de absorção no UV-Vis e cromatografia líquida de alta eficiência (CLAE), ambos com detecção na região do ultravioleta. ${ }^{5}$ Os métodos espectrofotométricos acoplados (ou não) aos sistemas cromatográficos fazem parte da maioria dos métodos citados na literatura para a determinação de NI, tanto em formulações farmacêuticas como em amostras biológicas, conforme relatado em revisão publicada recentemente. ${ }^{6}$ No entanto, grande parte dessas metodologias são de alto custo, requerem etapas complexas de pré-tratamento das amostras e geram resíduos provenientes da utilização de solventes orgânicos no procedimento analítico.

Os métodos eletroanalíticos apresentam características interessantes para serem explorados na determinação de uma diversidade de compostos em formulações farmacêuticas e amostras de composição química mais complexa, como fluidos biológicos e amostras

\footnotetext{
*e-mail: wallanst@ufvjm.edu.br
}

ambientais. Nesta perspectiva, o primeiro trabalho utilizando métodos eletroanalíticos para investigação do comportamento eletroquímico e determinação da NI foi descrito por Álvarez-Lueje e colaboradores. ${ }^{7}$ Esses autores utilizaram os eletrodos de trabalho de carbono vítreo e mercúrio (gotejante) para avaliar o comportamento eletroquímico da NI em função do $\mathrm{pH}$ na solução tampão Britton-Robinson em meio de água/etanol (70/30, v/v). A técnica de polarografia de pulso diferencial sobre o eletrodo gotejante de mercúrio em meio de $\mathrm{pH}$ 7,0 foi selecionada para determinação da NI em formulações farmacêuticas. Baseados em condições semelhantes (eletrólito e eletrodo de trabalho), outros autores descreveram a determinação de NI em fluidos biológicos, porém, um LD menor foi obtido mediante o uso da técnica de voltametria adsortiva de redissolução catódica por pulso diferencial sobre um eletrodo de gota pendente de mercúrio. ${ }^{8}$ Posteriormente, Catarino e co-autores ${ }^{9}$ propuseram a detecção amperométrica da NI sobre um eletrodo de carbono vítreo posicionado em um sistema FIA de duas linhas. Uma linha continha uma solução tampão que permitia a determinação de NI e a outra uma solução responsável pela limpeza e condicionamento da superfície do eletrodo de trabalho. O eletrodo de carbono vítreo foi novamente utilizado por outros autores na determinação da NI em amostras farmacêuticas,,$^{10}$ porém, a técnica eletroquímica empregada foi a voltametria adsortiva de redissolução catódica por varredura linear. Além disso, esse trabalho foi aplicado em amostras biológicas. Eletrodos modificados e de pasta de carbono também foram usados com sucesso na determinação de NI, tanto em amostras farmacêuticas quanto em fluidos biológicos. ${ }^{11}$ Outro método eletroanalítico reportado na literatura é baseado na potenciometria utilizando um eletrodo de membrana seletiva para determinação da NI. ${ }^{12}$

Nos últimos anos, eletrodos de diamante dopado com boro (BDD) vêm recebendo certo destaque em comparação aos demais eletrodos sólidos em função de suas caraterísticas, como: larga janela de potencial, baixa relação sinal/ruído, elevada relação corrente faradaica/corrente capacitiva e resistência à desativação e envenenamento durante a detecção de compostos eletroativos (fraca adsorção de moléculas em geral). ${ }^{13}$ Em consequência destas características, o eletrodo de 
BDD vem sendo amplamente utilizado na determinação de fármacos em diversas matrizes, ${ }^{14}$ assim como na degradação eletroquímica de poluentes emergentes. ${ }^{15}$

Recentemente, sistemas de análise por injeção em fluxo com detecção amperométrica de múltiplos pulsos (FIA-MPA, do inglês "flow injection analysis with multiple pulse amperometric detection") ${ }^{16}$ estão sendo explorados com sucesso em diversas aplicações. A possibilidade de aplicação de até 10 pulsos de potenciais em função do tempo e aquisição de até 10 amperogramas, independentes e simultâneos, possibilita a obtenção de vantagens em relação à amperometria convencional, como: (1) aumento na estabilidade de resposta de um eletrodo sólido em função do tempo (uso de pulso de potencial de limpeza): ${ }^{17}$ (2) aumento na seletividade do método (detecção do(s) produto(s) de oxidação ou redução) ${ }^{18}$ (3) possibilidade de determinações simultâneas em sistemas FIA $^{19}$ e BIA $^{20}$ empregando um único eletrodo de trabalho e; (4) implementação do método do padrão interno em sistemas FIA ${ }^{21}$ ou BIA ${ }^{22}$ com detecção amperométrica.

No presente trabalho apresentamos um novo método para determinação de NI por FIA-MPA em formulações farmacêuticas usando o eletrodo de BDD. O método proposto está baseado na combinação das características dos sistemas FIA-MPA e dos eletrodos de BDD para o desenvolvimento de uma análise simples, rápida e altamente estável, usando um eletrodo de trabalho sem a necessidade de modificação prévia de sua superfície.

\section{PARTE EXPERIMENTAL}

\section{Reagentes e soluções}

Os reagentes utilizados nos experimentos são de grau analítico e foram usados sem purificação prévia. As soluções foram preparadas em temperatura ambiente, utilizando água destilada e posteriormente deionizada (18,2 M $\Omega \mathrm{cm}$ ) obtida pelo sistema de purificação ELGA, modelo Di-MKZ. Uma solução estoque padrão de NI (Sigma) de $1,0 \times 10^{-2} \mathrm{~mol} \mathrm{~L}^{-1}$ foi preparada em etanol absoluto para as análises eletroquímicas e em meio de hidróxido de sódio $0,01 \mathrm{~mol} \mathrm{~L}^{-1}$ para as análises espectrofotométricas. Na detecção eletroquímica, as soluções padrão de trabalho foram obtidas por diluição em meio dos eletrólitos investigados. No caso da detecção espectrofotométrica, as soluções padrão de trabalho foram obtidas por diluição em hidróxido de sódio $0,01 \mathrm{~mol} \mathrm{~L}^{-1}$. As seguintes soluções de eletrólitos foram investigadas: ácido sulfúrico, tampão acetato $\mathrm{pH} 4,7$, tampão fosfato $\mathrm{pH}$ 7,0, tampão borato pH 9,2 e hidróxido de sódio. Todos os eletrólitos $(0,10$ mol $\mathrm{L}^{-1}$ ) foram preparados em água/etanol absoluto na proporção de 90:10, respectivamente.

As soluções tampão avaliadas foram preparadas da seguinte forma: (1) Em béqueres separados (contendo cerca de $800 \mathrm{~mL}$ de água) foram pesados uma massa correspondente a $0,1 \mathrm{~mol}$ de ácido acético, dihidrogênio fosfato de sódio $\left(\mathrm{H}_{2} \mathrm{PO}_{4}^{-}\right)$e ácido bórico; (2) Em cada béquer foi colocado um eletrodo de $\mathrm{pH}$, cujo $\mathrm{pHmetro}$ foi devidamente calibrado em soluções padrão de $\mathrm{pH}$ 7,0 e 4,0, para medidas em meio ácido, e em pH 7,0 e 10,0, para medidas em meio básico; (3) alíquotas de soluções de $\mathrm{NaOH} 1,0 \mathrm{~mol} \mathrm{~L}^{-1}$ foram adicionados em cada béquer até o $\mathrm{pH}$ estar exatamente em 4,7, 7,0 e 9,2 para as soluções tampão acetato, fosfato e borato, respectivamente; (4) As soluções tampão em cada béquer foram transferidas para um balão volumétrico e diluídas em água até o volume de 1,0 L.

Os compostos avaliados como potenciais interferentes foram os corantes tartrazina (Sigma), vermelho GRLX-220 (indústria têxtil de Divinópolis/MG), amarelo crepúsculo (Sigma), nova coccina (Sigma), e os fármacos paracetamol (Vetec), dipirona (Sigma), ácido ascórbico (Sigma), ácido salicílico (Vetec), ácido acetilsalicílico (Vetec), codeína (Sigma), piroxican (Sigma) e ibuprofeno (Sigma).

\section{Sistema FIA}

O sistema FIA utilizado neste estudo é de linha única, sendo composto basicamente por um sistema propulsor, um injetor, um detector eletroquímico e tubos de polietileno com 1,0 mm de diâmetro interno. A vazão do sistema FIA foi controlada por pressão de retorno gerada por uma coluna d'água e uma bomba de aquário. ${ }^{23}$

\section{Detecção eletroquímica}

O equipamento Potenciostato/Galvanostato da Autolab (Eco Chemie - modelo $128 \mathrm{~N}$ ) foi utilizado nos estudos voltamétricos e amperométricos. Uma célula eletroquímica convencional de três eletrodos foi utilizada nos estudos em sistema estacionário. No sistema em fluxo foi usada uma célula do tipo "wall jet" construída no próprio laboratório. ${ }^{24} \mathrm{Um}$ mini-eletrodo de referência de $\mathrm{Ag} / \mathrm{AgCl}\left(\mathrm{KCl}_{\mathrm{Sat}}\right)^{25}$ e um fio de platina foram utilizados como eletrodos de referência e auxiliar, respectivamente. Como eletrodo de trabalho foi utilizado um filme de diamante dopado com boro (BDD) em 8.000 ppm adquirido da empresa Adamant Technologies SA, La Chaux-de-Fonds, Suíça. A área geométrica utilizada do eletrodo BDD nas células foi de 7,0 $\mathrm{mm}^{2}$. Gandini e colaboradores descreveram os detalhes da elaboração do BDD. ${ }^{26} \mathrm{O}$ eletrodo de BDD foi inicialmente limpo em etanol e condicionado eletroquimicamente em meio de $\mathrm{H}_{2} \mathrm{SO}_{4} 0,5 \mathrm{~mol} \mathrm{~L}-1$ através de um tratamento catódico e anódico, aplicando -10,5 mA durante 60 s e $11,7 \mathrm{~mA}$ durante $30 \mathrm{~s}$, respectivamente. ${ }^{27} \mathrm{~A}$ avaliação da resposta eletroquímica do analito em função do tratamento do eletrodo de BDD foi realizada da seguinte forma: (1) o eletrodo foi condicionado de forma catódica, previamente, e em seguida foi realizado o tratamento anódico para investigação do comportamento eletroquímico da NI por meio da voltametria cíclico nos respectivos eletrólitos; (2) o procedimento inverso foi investigado para avaliar a resposta em função do tratamento catódico. Como não houve diferença significativa no perfil da resposta eletroquímica da NI após a avaliação dos tratamentos (catódico e anódico) sobre o BDD, o tratamento selecionado para limpeza da superfície do eletrodo foi o anódico, indicado no procedimento (1) acima. O procedimento de limpeza e tratamento eletroquímico do eletrodo era realizado diariamente e uma única vez antes do início dos experimentos. Todos os experimentos foram realizados sem a remoção do oxigênio dissolvido. Os dados dos gráficos apresentados foram tratados com o uso do software Origin 8.0. O cálculo do limite de detecção (LD) foi efetuado multiplicando o desvio padrão do branco $(n=10)$ por três e dividindo o resultado pelo coeficiente angular da curva de calibração.

\section{Preparo de amostras}

Formulações farmacêuticas de três marcas diferentes foram adquiridas em drogarias da cidade de Diamantina, estado de Minas Gerais, Brasil. Dez comprimidos de cada fabricante foram pesados e macerados. Em seguida, uma massa de aproximadamente 7,5 $\mathrm{mg}$ de cada amostra foi dissolvida em 10,0 mL de etanol usando um sonicador por 10 minutos. Após esta etapa, as soluções foram transferidas para um balão volumétrico e o volume completado com etanol para $25 \mathrm{~mL}$, constituindo a solução estoque da amostra. Antes das análises, alíquotas de cada solução estoque da amostra foram diluídas, adequadamente, na faixa linear de trabalho em meio do eletrólito suporte selecionado para as análises.

\section{Comparação do método por espectrofotometria de absorção no UV-Vis}

O procedimento de amostragem utilizado na análise FIA-MPA 
foi idêntico ao realizado por espectrofotometria de absorção no UVVis, método da Farmacopéia Brasileira. ${ }^{5}$ No entanto, a preparação da solução estoque da amostra foi realizada pela adição de uma massa de 7,5 mg da amostra em $50 \mathrm{~mL}$ de $\mathrm{NaOH} 0,01 \mathrm{~mol} \mathrm{~L}^{-1}$. A solução obtida foi previamente filtrada e transferida para um balão volumétrico, sendo o volume completado com $\mathrm{NaOH} 0,01 \mathrm{~mol} \mathrm{~L}^{-1}$ para $250,0 \mathrm{~mL}$. As soluções padrão de NI $\left(5,0 \times 10^{-5}\right.$ a 5,0 x $\left.10^{-4} \mathrm{~mol} \mathrm{~L}^{-1}\right)$ foram preparadas em meio de $\mathrm{NaOH} 0,01 \mathrm{~mol} \mathrm{~L}^{-1}$ para construção da curva de calibração. O comprimento de onda foi fixado em $392 \mathrm{~nm}$.

\section{RESULTADOS E DISCUSSÕES}

\section{Otimização do método analítico}

O comportamento eletroquímico da NI foi investigado por voltametria cíclica em função da natureza do eletrólito suporte sobre o eletrodo de BDD. As melhores condições eletroquímicas em função da sensibilidade e seletividade na detecção da NI foram obtidas em meio de tampão fosfato $0,1 \mathrm{~mol} \mathrm{~L}^{-1}(\mathrm{pH} 7,0)$, preparado em água e posteriormente misturado com etanol na proporção 90:10, respectivamente. Os voltamogramas cíclicos deste estudo são apresentados na Figura 1.

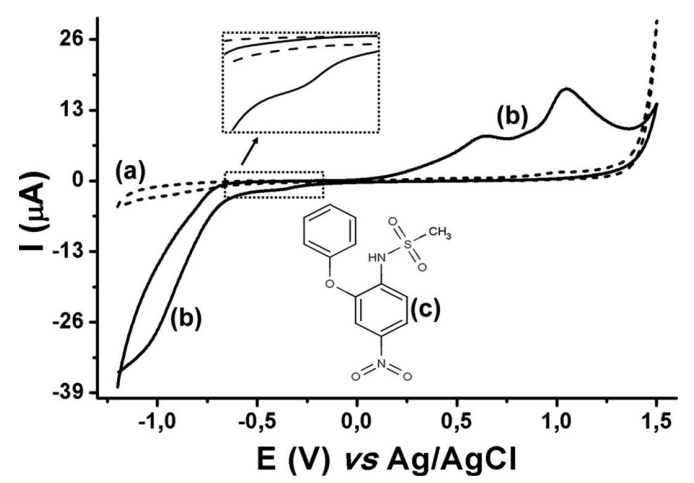

Figura 1. Voltamogramos cíclicos obtidos com eletrodo de BDD em meio de tampão fosfato 0,1 mol $L^{-1}$ (pH 7,0) e etanol (90:10) sem (a) e com (b) adição de NI 5,0 $\times 10^{-3} \mathrm{~mol} \mathrm{~L}^{-1}$. Velocidade de varredura: $50 \mathrm{mV} \mathrm{s}^{-1}$; A varredura cíclica de potencial foi iniciada em -1,2 V vs Ag/AgCl. (c) Estrutura química da NI. Em detalhe, o processo de redução do grupo nitroso gerado pelo processo de oxidação em 0,6 V da NI

Como pode ser observado na Figura 1, a NI apresenta dois processos de oxidação $(0,60$ e 1,05 V) e dois de redução $(-0,40$ e $-1,00 \mathrm{~V})$ sobre o eletrodo de BDD em meio de tampão fosfato $(\mathrm{pH}$ 7,0 ). Os processos eletroquímicos que ocorrem sobre o eletrodo de BDD são semelhantes aos descritos na literatura quando mercúrio e carbono vítreo foram usados como eletrodos de trabalho em $\mathrm{pH} 7,5 .^{7}$ O segundo pico de oxidação da NI $(1,05 \mathrm{~V})$ provavelmente está relacionado com uma oxidação irreversível do grupo metilsulfonamida presente em sua estrutura. ${ }^{7}$ Os outros processos eletroquímicos estão relacionados com a presença do grupo nitro na estrutura da NI. ${ }^{7}$ Inicialmente, o grupo nitro $\left(-\mathrm{NO}_{2}\right)$ é eletroquimicamente reduzido de forma irreversível (o processo inicia em torno de $-0,60 \mathrm{~V}$ ), gerando o grupo hidroxilamina (-NHOH), mais quatro elétrons e quatro íons hidrogênio. Posteriormente, seguindo a varredura em direção a potenciais mais positivos, o grupo $-\mathrm{NHOH}$ é oxidado ao grupo nitroso (-NO) de forma quase-reversível (este processo inicia em torno de $0,20 \mathrm{~V})$, liberando dois elétrons e dois íons hidrogênio. Na varredura inversa, o grupo -NO é reduzido gerando novamente o grupo - $\mathrm{NHOH}$ de forma quase-reversível (o processo inicia em torno de -0,30 V). Cabe ressaltar que o processo de oxidação em $0,6 \mathrm{~V}$ e o de redução antes de $-0,6 \mathrm{~V}$ somente existem se a NI for previamente reduzida em potenciais mais negativos do que $-0,6 \mathrm{~V}$.

Caso a amperometria convencional (potencial constante) fosse utilizada na quantificação de NI usando um sistema FIA, duas opções poderiam ser utilizadas: (1) aplicação de um potencial negativo (menor que -0,6 V) e redução do grupo nitro ou (2) aplicação de um potencial positivo (maior que $0,8 \mathrm{~V}$ ) e oxidação do grupo metilsulfonamida. Nestas condições, qualquer espécie que for reduzida $(\leq-0,6 \mathrm{~V})$ ou oxidada $(\geq 0,8 \mathrm{~V})$ nesta mesma região de potencial pode ser considerada um interferente na análise por meio da detecção amperométrica. Além disto, empregando amperometria convencional, nenhuma informação adicional é fornecida. Em função disto, estamos propondo neste trabalho o uso da FIA-MPA, aplicando três pulsos de potenciais para monitoramento dos três processos eletroquímicos relacionados ao grupo nitro da NI. Desta forma, informações adicionais podem ser adquiridas, por meio de uma simples injeção de uma alíquota de solução amostra, no intuito de aumentar a seletividade do método para quantificação da NI.

A seleção dos pulsos de potenciais e seus respectivos tempos de aplicação foram avaliados em função da seletividade e sensibilidade do método para quantificação de NI. Nestes estudos, a alça de amostragem e a vazão do sistema FIA-MPA foram mantidas fixas em $100 \mu \mathrm{L}$ e 2,0 $\mathrm{mL} \mathrm{min}^{-1}$, respectivamente. Apesar do eletrodo de BDD apresentar boa resistência, após alguns estudos iniciais foi verificado que havia adsorção ou envenenamento de sua superfície, sendo necessária a aplicação de um quarto pulso de potencial. Este potencial tem a função de efetuar a constante limpeza eletroquímica da superfície do eletrodo de BDD e aumentar a estabilidade (repetibilidade) do sistema.

Após os estudos de otimização, a seguinte sequência foi selecionada para aplicação dos pulsos de potenciais e seus respectivos tempos de duração:

(1) $\mathrm{E}_{1(\mathrm{det})}=-0,80 \mathrm{~V} / 30 \mathrm{~ms}$ : redução do grupo $\mathrm{NO}_{2}$ da NI e geração do grupo $\mathrm{NHOH}$

(2) $\mathrm{E}_{2 \text { (det) }}=0,60 \mathrm{~V} / 30 \mathrm{~ms}$ : oxidação do grupo $\mathrm{NHOH}$ gerado no pulso de potencial anterior e geração de $\mathrm{NO}$;

(3) $\mathrm{E}_{3(\mathrm{det})}=-0,40 \mathrm{~V} / 30 \mathrm{~ms}$ : redução do grupo $\mathrm{NO}$ gerado no pulso de potencial anterior;

(4) $\mathrm{E}_{4(\text { limp) }}=-0,45 \mathrm{~V} / 300 \mathrm{~ms}$ : limpeza eletroquímica da superfície do eletrodo de BDD e obtenção de melhor seletividade.

A aplicação desta sequência de pulsos de potenciais permite a aquisição de quatro amperogramas independentes por meio do software que controla o potenciostato (GPES 4.9 - Metrohm Autolab). Cabe destacar que o objetivo da aplicação do pulso de potencial de limpeza em - $0,45 \mathrm{~V}$ durante $300 \mathrm{~ms}$ também está relacionado à seletividade do método para detecção da NI. Isso porque, quando o pulso de potencial de limpeza em $-0,45 \mathrm{~V}$ por $300 \mathrm{~ms}$ não era aplicado, os produtos de oxidação (gerados em $0,6 \mathrm{~V}$ ) de espécies como, por exemplo, paracetamol e dipirona, são reduzidos em $-0,8 \mathrm{~V}$ e, portanto, podem ser considerados interferentes na determinação de NI com o método proposto. Na aplicação do pulso de potencial em $-0,45 \mathrm{~V}$ por $300 \mathrm{~ms}$, os produtos de oxidação do paracetamol e dipirona são reduzidos ou consumidos e não há detecção de corrente no pulso de potencial de $-0,8 \mathrm{~V}$. Assim, pode haver uma melhora significativa na seletividade do método proposto. Este assunto é discutido com mais detalhes no final deste trabalho. Outra opção seria a aplicação de um pulso de potencial na região de desprendimento de oxigênio para renovação (limpeza) da superfície do eletrodo de BDD. No entanto, em sistemas FIA, isto não é recomendável, pois o surgimento de bolhas no sistema pode comprometer a análise. Por fim, normalmente, opta-se por não registrar a corrente detectada no pulso de potencial responsável pela limpeza eletroquímica do eletrodo de trabalho (-0,45 V / $300 \mathrm{~ms})$.

Em seguida, aplicando esta sequencia de pulsos de potenciais, os 
parâmetros referentes ao volume de amostragem e vazão da solução carregadora foram investigados. Em ambos os casos, a seleção foi feita em função da altura de pico (corrente) detectado em cada pulso de potencial referente à injeção de uma solução de NI $1,0 \times 10^{-4} \mathrm{~mol} \mathrm{~L}^{-1}$. As melhores condições foram obtidas em volume de amostragem de $200 \mu \mathrm{L}$ e vazão do sistema de 3,6 $\mathrm{mL} \mathrm{min}^{-1}$. Maiores detalhes sobre a influência desses parâmetros sobre a resposta obtida por FIA-MPA podem ser verificados no artigo de revisão publicado, recentemente, por dos Santos e colaboradores. ${ }^{16}$

\section{Estudos dos parâmetros analíticos}

Na Figura 2 são apresentados os resultados obtidos no estudo de repetibilidade do sinal analítico mediante injeções sucessivas $(\mathrm{n}=$ 10) de uma solução contendo NI $1,0 \times 10^{-4} \mathrm{~mol} \mathrm{~L}^{-1}$.

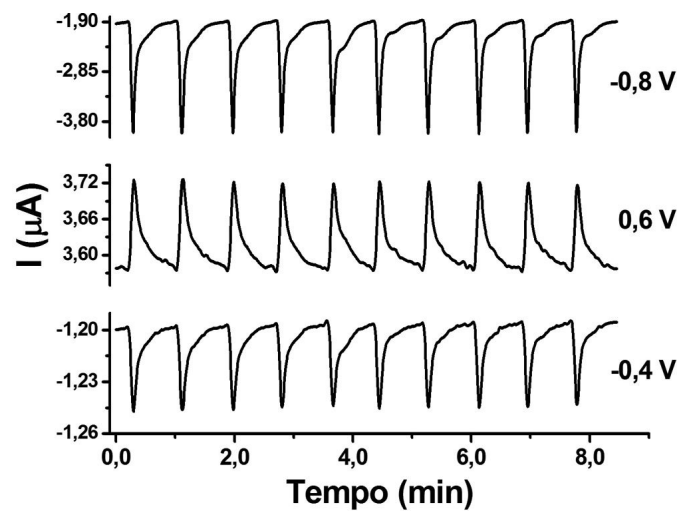

Figura 2. Amperogramas obtidos por FIA-MPA de 10 injeções sucessivas de NI 1,0 × $10^{-4} \mathrm{~mol} \mathrm{~L}^{-1}$. Pulsos de potenciais aplicados: -0,8 V/30 ms, 0,6 V/30 ms e-0,4 V/30 ms; pulso de potencial de limpeza: -0,45V/300 ms (amperograma não apresentado); vazão de 3,6 $\mathrm{mL} \mathrm{min}^{-1}$; volume injetado: $200 \mu \mathrm{L}$

A partir dos resultados apresentados na Figura 2, o desvio padrão relativo (RSD) foi calculado para cada amperograma adquirido nos pulsos de potenciais de $-0,8,0,6,-0,4 \mathrm{~V}$, obtendo-se o valor de $0,33 \%$, $0,28 \%$ e $0,11 \%$, respectivamente. Estes resultados demonstram que o método proposto apresenta melhor desempenho (RSD $50 \%$ menor) quando comparado aos métodos eletroquímicos publicados anteriormente para determinação de NI. ${ }^{7-12}$ Vale lembrar que, apesar da alta estabilidade do eletrodo de BDD, a limpeza eletroquímica (obtida pela aplicação do pulso de potencial em $-0,45 \mathrm{~V} / 300 \mathrm{~ms}$ ) durante as análises foi fundamental para que houvesse uma resposta altamente estável nos pulsos de potenciais aplicados. Nessas condições, a frequência analítica foi calculada em torno de 90 determinações por hora, cujo valor é quase o dobro em relação ao método FIA descrito na literatura para determinação de NI. ${ }^{9}$

A faixa linear de trabalho foi determinada em função de cada pulso de potencial utilizado no sistema FIA-MPA proposto, sendo que os resultados obtidos foram os seguintes: $2,0 \times 10^{-7}$ a $8,0 \times 10^{-5}$ mol L-1 em $-0,8 \mathrm{~V} ; 6,0 \times 10^{-7}$ a $8,0 \times 10^{-5} \mathrm{~mol} \mathrm{~L}^{-1}$ em $0,6 \mathrm{~V}$; e $4,0 \times 10^{-6}$ a $8,0 \times 10^{-5} \mathrm{em}-0,4 \mathrm{~V}$. Os coeficientes de correlação linear obtidos em todos os pulsos de potenciais foram superiores a 0,99. Os limites de detecção foram calculados em 0,081, 0,103 e 3,470 $\mu \mathrm{mol} \mathrm{L}^{-1}$ para os pulsos de potenciais de $-0,8,0,6$ e $-0,4 \mathrm{~V}$, respectivamente. A faixa linear de trabalho foi limitada entre 4,0 × $10^{-6}$ a $8,0 \times 10^{-5} \mathrm{~mol} \mathrm{~L}^{-1}$ para a determinação da NI empregando os três pulsos de potenciais selecionados. Na Figura 3 são apresentados os amperogramas e as suas respectivas curvas de calibração obtidas nos três pulsos de potenciais após a injeção de soluções padrão contendo concentrações de NI.

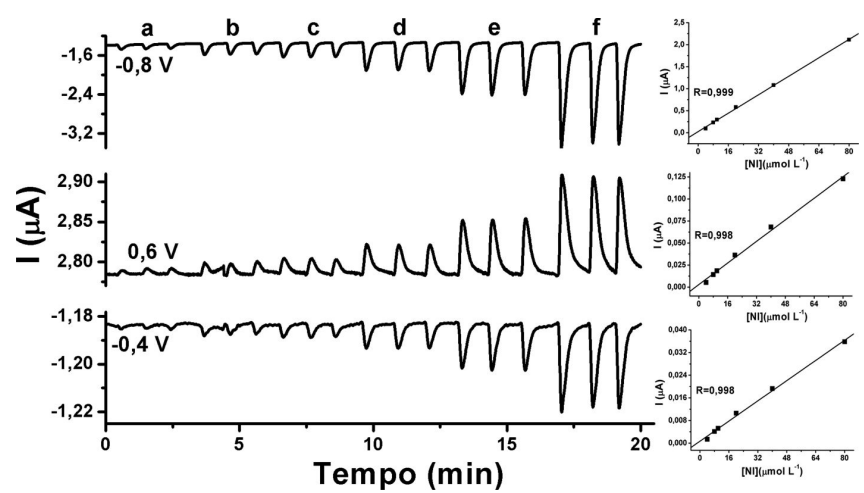

Figura 3. FIAgramas e respectivas curvas de calibração obtidos por FIA-MPA sobre o eletrodo de BDD com a injeção de soluções padrão contendo 4,0 (a), 8,0 (b), 10,0 (c), 20,0 (d), 40,0 (e) e 80,0 (f) $\mu \mathrm{mol} L^{-1}$ de NI. Demais parâmetros: conforme Figura 2

As equações 1, 2 e 3 foram obtidas a partir das regressões lineares das curvas de calibração apresentadas na Figura 3.

$\mathrm{I}(\mu \mathrm{A})=0,02339+0,02624[\mathrm{NI}]\left(\mu \mathrm{mol} \mathrm{L} \mathrm{L}^{-1}\right)$, para $-0,8 \mathrm{~V}$

$\mathrm{I}(\mu \mathrm{A})=0,00289+0,00153[\mathrm{NI}](\mu \mathrm{mol} \mathrm{L}-1)$, para $0,6 \mathrm{~V}$

$\mathrm{I}(\mu \mathrm{A})=0,00068+0,000446[\mathrm{NI}]\left(\mu \mathrm{mol} \mathrm{L} \mathrm{L}^{-1}\right)$, para $-0,4 \mathrm{~V}$

O desempenho do método proposto foi avaliado na análise de 3 amostras de formulações farmacêuticas contendo NI. Os estudos de adição e recuperação de soluções padrão de NI na amostra também foram investigados. Na Tabela 1 são apresentados os resultados obtidos

Tabela 1. Estudos de adição e recuperação em amostras farmacêuticas e determinação de NI por espectrofotometria (método da farmacopeia) e pelo método proposto (FIA-MPA)

\begin{tabular}{|c|c|c|c|c|c|}
\hline $\begin{array}{c}\text { Amostras de NI } \\
\text { (100 mg / comprimido) }\end{array}$ & $\begin{array}{l}\text { Adição de } \\
\text { NI (mg) }\end{array}$ & $\begin{array}{l}\text { Esperado de } \\
\text { NI (mg) }\end{array}$ & $\begin{array}{c}\text { Determinação de NI } \\
\text { por MPA-FIA* } \\
(\mathrm{mg})\end{array}$ & $\begin{array}{c}\text { Recuperação da NI } \\
\text { por MPA-FIA* } \\
(\%)\end{array}$ & $\begin{array}{l}\text { Determinação de NI } \\
\text { por espectrofotometria } \\
(\mathrm{mg})\end{array}$ \\
\hline \multirow{3}{*}{ A } & 0,0 & 100,0 & $103,5 \pm 0,6$ & --- & $104,0 \pm 0,8$ \\
\hline & 10,0 & 113,5 & $115,9 \pm 0,6$ & 101,8 & -- \\
\hline & 30,0 & 133,5 & $132,3 \pm 0,9$ & 99,1 & --- \\
\hline \multirow{3}{*}{ B } & 0,0 & 100,0 & $104,2 \pm 0,6$ & -- & $103,6 \pm 0,6$ \\
\hline & 10,0 & 104,2 & $107,6 \pm 0,7$ & 103,3 & --- \\
\hline & 30,0 & 134,2 & $135,1 \pm 0,9$ & 100,7 & --- \\
\hline \multirow{3}{*}{$\mathrm{C}$} & 0,0 & 100,0 & $104,2 \pm 0,5$ & --- & $105,6 \pm 0,6$ \\
\hline & 10,0 & 114,2 & $112,2 \pm 0,6$ & 98,2 & --- \\
\hline & 30,0 & 134,2 & $133,8 \pm 0,9$ & 99,7 & --- \\
\hline
\end{tabular}

"Os resultados (média \pm DP; $n=3$ ) obtidos por meio da MPA são relacionados ao pulso de potencial em $-0,8 \mathrm{~V}$. 
Tabela 2. Comparação dos parâmetros analíticos obtidos pelo método proposto e outros métodos eletroanalíticos descritos na literatura para determinação de NI

\begin{tabular}{|c|c|c|c|c|c|}
\hline Técnica Eletroanalítica* & Eletrodo de trabalho** & $\begin{array}{l}\text { Faixa linear } \\
\mu \mathrm{mol} \mathrm{L} \mathrm{L}^{-1}\end{array}$ & $\begin{array}{c}\text { LD } \\
\mu \mathrm{mol} \mathrm{L} \mathrm{L}^{-1}\end{array}$ & $\begin{array}{c}\mathrm{RSD}^{* * * *} \\
(\%)\end{array}$ & Aplicação em Amostra \\
\hline $\mathrm{PPD}^{7}$ & DME & 5,0 a 50 & 2,5 & 0,36 & Farmacêutica \\
\hline VRCPD $^{8}$ & HDME & 0,0030 a 0,30 & 0,0013 & 1,3 & Biológica \\
\hline ACFIA $^{9}$ & GCE & 50 a 300 & 3,1 & 1,1 & Farmacêutica \\
\hline VRCVL $^{10}$ & GCE & 0,40 a 50 & 0,032 & 0,61 a 1,46 & Farm. e Biol. \\
\hline $\mathrm{VVL}^{11}$ & MWCNTs/GCE & 0,32 a 65 & 0,16 & 3,6 & Farmacêutica \\
\hline $\mathrm{VPD}^{11}$ & $\mathrm{Ac} / \mathrm{CNTs} / \mathrm{GCE}$ & 0,10 a 10 & 0,050 & 2,9 & Farm. e Biol. \\
\hline $\mathrm{VPD}^{11}$ & SiC-NPs/GCE & 0,090 a 8,7 & 0,030 & -- & Biológica \\
\hline $\mathrm{VPD}^{11}$ & EPC & 0,50 a 10 & 0,0086 & 1,02 & Farm. Biol. \\
\hline $\mathrm{POT}^{12}$ & NIM & 1,0 a 10000 & 0,10 & --- & Farmacêutica \\
\hline FIA-MPA & BDD & 0,20 a 80 & 0,081 & 0,11 a 0,30 & Farmacêutica \\
\hline
\end{tabular}

$*$ PPD = polarografia de pulso diferencial; VRCPD = voltametria de redissolução catódica por pulso diferencial; ACFIA = amperometria convencional em sistema FIA; VRCVL = voltametria de redissolução catódica por varredura linear; VVL voltametria de varredura linear; VPD = voltametria de pulso diferencial; $\mathrm{POT}=$ potenciometria; $* * \mathrm{DME}=$ eletrodo gotejante de mercúrio; HDME = eletrodo de gota pendente de mercúrio; GCE = eletrodo de carbono vítreo; MWCNTs/GCE = eletrodo de carbono vítreo modificado com nanotubos de carbono de paredes múltiplas; Ac/CNTs/GCE = eletrodo de carbono vítreo modificado com nanotubos de carbono e Ácido cistéico; $\mathrm{SiC}-\mathrm{NPs} / \mathrm{GCE}=$ eletrodo de carbono vítreo modificado com nanoparticulas de $\mathrm{SiC} ; \mathrm{EPC}=\mathrm{Eletrodo}$ de pasta de carbono; NIM = eletrodo de membrana seletivo a Nimesulida; ***O número de medidas das técnicas foram entre 9 e 12 , exceto para a VRCVL e VPD (usando EPC), que foram de 5 medidas. Os estudos de repetibilidade foram considerados para um mesmo eletrodo de trabalho, porém as concentrações de NI foram diferentes em cada artigo.

nos estudos de adição e recuperação, bem como da comparação dos resultados obtidos pelo método proposto frente ao método descrito na Farmacopeia Brasileira (espectrofotometria). ${ }^{5}$ Os resultados apresentados na Tabela 1 foram obtidos por meio da curva de calibração para o pulso de potencial aplicado em - $0,8 \mathrm{~V}$. Nos demais pulsos de potenciais, os resultados foram similares e podem ser verificados em material suplementar (Tabelas $1 \mathrm{~S}$ e $2 \mathrm{~S}$ ).

Conforme os resultados apresentados na Tabela 1, os estudos de adição e recuperação foram próximos de $100 \%$, indicando ausência de efeitos de matriz nestas amostras para a determinação de NI. O test $t$ foi aplicado nos resultados obtidos para cada amostra empregando-se os dois métodos analíticos. Os resultados indicaram que não há diferença significativa entre os dois métodos com nível de confiança de $95 \%$. Além disso, pode ser verificado que não há praticamente diferença na quantidade de NI encontrada nas amostras em relação ao valor rotulado nas formulações farmacêuticas analisadas. Na Tabela 2 são apresentados os parâmetros analíticos obtidos pelo método proposto e para os demais métodos eletroanalitícos descritos na literatura para determinação de NI.

A avaliação da Tabela 2 mostra que os parâmetros analíticos obtidos por meio do método proposto para determinação de NI estão próximos aos valores dos demais métodos eletroanalíticos relatados neste trabalho. Como destaque, a repetibilidade do método FIA-MPA utilizando o eletrodo de BDD apresentou um valor inferior frente aos métodos citados, principalmente, em relação aos eletrodos de trabalho modificados. No que tange a comparação entre outras técnicas, baseado na revisão publicada recentemente para determinação de NI, ${ }^{6}$ os resultados apresentados pelo método proposto foram próximos ou melhores em relação aos demais, por exemplo, um menor LD em relação à maioria das técnicas espectrofotométricas. No entanto, há alguns trabalhos nesta revisão que possuem larga faixa linear de trabalho e LDs menores que $1,0 \times 10^{-9} \mathrm{~mol} \mathrm{~L}^{-1}$.

\section{Estudos para aumentar a seletividade na detecção de NI em amostras farmacêuticas e ambientais}

Outro ponto a ser destacado nos resultados apresentados na Figura 3 é a razão existente entre os sinais amperométricos detectados entre os três pulsos de potenciais. Na Tabela 3 são apresentadas as razões médias entre a corrente detectada na faixa linear de trabalho avaliada na Figura 3.

Tabela 3. Razões existentes entre as correntes detectadas para NI nos pulsos de potenciais aplicados sobre o eletrodo de BDD

\begin{tabular}{ccc}
\hline Pulsos de potenciais & Razão \pm DP & RSD $(\%)$ \\
\hline$-0,8 \mathrm{~V} / 0,6 \mathrm{~V}$ & $16,3 \pm 0,5$ & 3 \\
$-0,8 \mathrm{~V} /-0,4 \mathrm{~V}$ & $56,5 \pm 1,6$ & 2,8 \\
$0,6 \mathrm{~V} /-0,4 \mathrm{~V}$ & $3,48 \pm 0,05$ & 1 \\
\hline
\end{tabular}

Como pode ser observado, o RSD foi igual ou inferior a $3 \%$ nas três relações avaliadas, demonstrando que a razão entre a corrente detectada nos três pulsos de potenciais é constante e independe da concentração. Esta avaliação é importante e permite obter informações para aumentar a seletividade da detecção de NI em amostras farmacêuticas e ambientais. Isso porque, caso estes valores (razões) forem encontrados para soluções amostra, há grande possibilidade de que a NI esteja presente sem interferentes eletroativos nos pulsos de potenciais aplicados na solução amostra por meio da FIA-MPA. Apesar de não se tratar de uma conclusão definitiva da presença de NI, a detecção por MPA pode ser utilizada como uma triagem ("screening") rápida e de baixo custo para uma posterior quantificação mais exata por outra técnica analítica. Quando uma razão diferente for detectada, o método proposto não poderá ser usado na quantificação de NI, pois é um indício da presença de outro composto eletroativo. Cabe salientar que, apesar da amperometria convencional (potencial constante) ser mais simples, este modo de detecção não permitiria a obtenção dessa informação. Não obstante, as técnicas como voltametria cíclica, voltametria de pulso diferencial e voltametria de onda quadrada também permitiriam a obtenção de informações similares ao método proposto. No entanto, as técnicas voltamétricas podem apresentar uma frequência analítica muito menor frente ao FIA-MPA. Além disso, uma atenção especial deveria ser dada à contaminação da superfície do eletrodo de BDD, pois as técnicas voltamétricas não permitem a inclusão de uma etapa de limpeza eletroquímica durante o procedimento de análise. 
Alguns possíveis interferentes classificados como poluentes emergentes em águas residuais foram avaliados pelo método proposto. $\mathrm{Na}$ Figura 4 são apresentados os resultados obtidos pelo sistema proposto mediante a injeção em triplicata de soluções contendo os seguintes compostos: tartrazina, vermelho GRLX-220, amarelo crepúsculo, paracetamol, ácido salicílico, dipirona, nova coccina, codeína, ácido ascórbico, ácido acetilsalicílico, ibuprofeno e piroxicam.

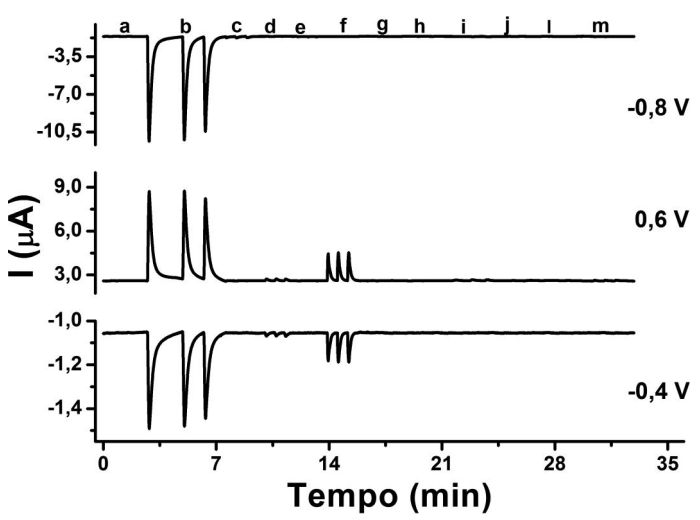

Figura 4. Amperogramas obtidos pela MPA em FIA sobre BDD para avaliação dos interferentes na detecção de NI em amostras ambientais. Os interferentes foram injetados em triplicata na seguinte ordem: (a) tartrazina, (b) vermelho GRLX-220 (c) amarelo crepúsculo, (d) paracetamol, (e) ácido salicílico, (f) dipirona, $(\boldsymbol{g})$ nova coccina, $(\boldsymbol{h})$ codeína, $(\boldsymbol{i})$ ácido ascórbico, $(\boldsymbol{j})$ ácido acetilsalicílico, $(\boldsymbol{l})$ ibuprofeno $e(\boldsymbol{m})$ piroxicam. Todos nas concentrações de 1,0 $\times 10^{-4} \mathrm{~mol} \mathrm{~L}^{-1}$. Os parâmetros da detecção MPA e do sistema FIA forma os mesmos da Figura 2

Como pode ser observado na Figura 4, muitos compostos como a tartrazina, ácidos salicílico, ascórbico e acetilsalicílico, nova coccina, codeína, ibuprofeno e piroxican não seriam potenciais interferentes para determinação da NI em amostras ambientais e farmacêuticas. No entanto, na presença dos compostos como amarelo crepúsculo, paracetamol e dipirona, nas condições estabelecidas para análise de NI, haveria uma interferência na detecção em alguns dos pulsos de potenciais monitorados. No caso da presença do composto amarelo crepúsculo, há uma pequena interferência na corrente detectada no pulso de potencial de $-0,8 \mathrm{~V}$, porém esta interferência não é observada nos demais pulsos e a NI poderia continuar sendo seletivamente quantificada pelo método proposto. Outros dois compostos interferentes na quantificação da NI seriam o paracetamol e a dipirona. Neste caso, a interferência ocorre nos pulsos de potenciais de 0,6 e $-0,4 \mathrm{~V}$, mas a NI ainda poderia ser determinada com a corrente detectada no pulso de potencial de $-0,8$ V. Cabe ressaltar que, o processo de redução observado em $-0,4 \mathrm{~V}$ para dipirona e paracetamol não foi verificado em $-0,8 \mathrm{~V}$ devido à aplicação do pulso de potencial de limpeza em -0,45 V durante 300 ms. Apenas no caso da interferência acontecer simultaneamente nos três pulsos de potenciais aplicados, a NI não poderia ser quantificada seletivamente por este método. Este fenômeno foi observado na solução contendo o corante vermelho GRLX-220 (eletroativo nos três pulsos de potenciais). No entanto, as relações calculadas entre os picos de correntes para este composto nos três pulsos de potenciais são diferentes $(-0,8 \mathrm{~V} / 0,6 \mathrm{~V}=1,2 ;-0,8 \mathrm{~V} /-0,4 \mathrm{~V}=1,1$ e $0,6 \mathrm{~V} /-0,4 \mathrm{~V}=0,95)$ dos valores calculados para NI $(16,3 ; 56,5$ e 3,48 ), respectivamente. Portanto, o método proposto permite perceber facilmente que o composto detectado não é apenas NI. Neste caso, a determinação de NI somente seria possível se um método de separação prévio ou em linha (cromatografia) fosse empregado.

Todavia, pequenas variações podem ocorrer nas razões calculadas entre os picos de correntes nos três pulsos de potenciais monitorados (Tabela 3). Este fenômeno pode ocorrer em função de pequenas alterações no procedimento de limpeza eletroquímica do eletrodo de BDD ou de mudanças nos parâmetros do sistema FIA, principalmente, na vazão. Contudo, se os parâmetros do sistema FIA e o procedimento de limpeza eletroquímica do eletrodo forem mantidos inalterados, a razão calculada entre os picos de correntes nos três pulsos de potenciais monitorados será constante. Uma forma de amenizar esta dependência é sempre incluir no procedimento a injeção de uma solução padrão contendo a NI. Assim, no momento da injeção essas razões entre os sinais seriam recalculadas, evitando um possível erro na avaliação do método proposto.

\section{MATERIAL SUPLEMENTAR}

As Tabelas 1S e 2S estão disponíveis em http://quimicanova.sbq. org.br, em arquivo pdf, com acesso livre.

\section{CONCLUSÕES}

O presente trabalho apresentou um método mais simples e rápido frente aos demais métodos descritos para quantificação da NI em formulações farmacêuticas. Aliada a essas vantagens, o método proposto por meio da FIA-MPA sobre o eletrodo de trabalho de BDD proporcionou uma alta sensibilidade, seletividade e reprodutibilidade sem a necessidade de modificação do eletrodo de trabalho. Dessa forma, este método pode ser uma alternativa extremamente atrativa para o controle de qualidade de rotina da NI em laboratórios e indústrias farmacêuticas. Além disso, a possibilidade do monitoramento de vários sinais amperométricos simultaneamente pela detecção MPA pode aumentar a seletividade para determinação de NI em amostras mais complexas, como em águas e fluidos biológicos.

\section{AGRADECIMENTOS}

Ao Conselho Nacional de Desenvolvimento Científico e Tecnológico (CNPq), à Coordenação de Aperfeiçoamento de Pessoal de Nível Superior (CAPES), à Rede Mineira de Química e à Fundação de Amparo à Pesquisa do Estado de Minas Gerais (FAPEMIG) pelo suporte financeiro.

\section{REFERÊNCIAS}

1. Goodman, L.; Gilman, A. Em Goodman \& Gilman's The Pharmacological Basis of Therapeutics; Brunton, L. L.; Lazo, J. S.; Parker, K. L., Eds.; McGraw Hill: Nova Iorque, 2006, cap. 24.

2. Rabasseda, X.; Drugs Today 1997, 33, 41.

3. http://pfarma.com.br/noticia-setor-farmaceutico/industriafarmaceutica/876-10- medicamentos-genericos-mais-consumidos-2011. html, acessado em Dezembro 2012.

4. da Silva, C. G. A.; Collins, C. H.; Quim. Nova 2011, 34, 665.

5. Agência Nacional de Vigilância Sanitária (ANVISA); Farmacopeia Brasileira, 5 ${ }^{\text {a }}$ Ed., ANVISA: Brasília, 2010.

6. Starek, M.; Krzek, J.; Talanta 2009, 77, 925.

7. Álvarez-Lueje, A,; Váquez, P.; Núñez-Vergata, L. J.; Squella, J. A.; Electroanalysis 1997, 9, 1209.

8. Furlanetto, S.; Orlandini, S.; Aldini, G.; Gotti, R.; Dreassi, E.; Pinzauti, S.; Anal. Chim. Acta 2000, 413, 229.

9. Catarino, R. I. L.; Conceição, A. C. L.; Garcia, M. B. Q.; Gonçalves, M. L. S.; Lima, J. L. F. C.; dos Santos, M. M. C.; J. Pharm. Biomed. Anal. 2003, 33, 571.

10. El-Sayed, G. O.; Yasin, S. A.; El-Ries, M. A.; El-Badawy, A. A.; Lat. Am. J. Pharm. 2009, 28, 741. 
11. Zhang, J.; Tan, X.; Zhao, D.; Tan, S.; Huang, Z.; Mi, Y.; Huang, Z.; Electrochim. Acta 2010, 55, 2522; Wang, C.; Shao, X.; Liu, Q.; Qu, Q.; Yang, G.; Hu, X.; J. Pharm. Biomed. Anal. 2006, 42, 237; Ghavami, R.; Navaee, A.; Microchim. Acta 2012, 176, 493. Malode, S. J.; Nandibewoor, S. T.; Z. Phys. Chem. 2013, 227, 73.

12. Kumar, K. G.; Augustine, P.; John, S.; Port. Electrochim. Acta 2007, 25 , 375.

13. de Barros, R. C. M.; Ribeiro, M. C.; An-Sumodjo, P. T.; Julião, M. S. S.; Serrano, S. H. P.; Ferreira, N. G.; Quim. Nova 2005, 28, 317. Pleskov, Y.V.; J. Anal. Chem. 2000, 55, 1045. Suffredini, H. B.; Pedrosa, V. A.; Codognoto, L.; Machado, S. A. S.; Rocha-Filho, R. C.; Avaca, L. A.; Electrochim. Acta 2004, 49, 4021. Swain, G. M. Em Electroanalytical Chemistry; Bard, A. J.; Rubinstein, I., eds.; Marcel Dekker: Nova Iorque, 2004, vol. 22.

14. Lopes, A. C. V.; Luz, R. C. S.; Damos, F. S.; dos Santos, A. S.; Franco, D. L.; dos Santos W. T. P.; J. Braz. Chem. Soc. 2012, 23, 1800; Sartori, E. R.; Medeiros, R. A.; Rocha, R. C.; Fatibello-Filho, O.; J. Braz. Chem. Soc. 2009, 20, 360; Ribeiro, F. W. P.; Cardoso, A. S.; Portela, R. R.; Lima, J. E. S.; Machado, S. A. S.; De Lima, P.; De Souza, D.; Correia, A. N.; Electroanalysis 2008, 20, 2031.

15. Sirés, I.; Brillas, E.; Environ. Int. 2012, 40, 212.

16. dos Santos, W. T. P.; Gimenes, D. T.; Richter, E. M.; Angnes, L.; Quim. Nova 2011, 34, 1753.

17. Gimenes, D. T.; de Freitas, J. M.; Munoz, R. A. A.; Richter, E. M.; Electroanalysis 2011, 23, 2521; Stefano, J. S.; de Lima, A. P.; Montes, R. H. O.; Richter, E. M.; Munoz, R. A. A.; J. Braz. Chem. Soc. 2012, 23, 1834.
18. dos Santos, W. T. P.; Gimenes, D. T.; de Almeida, E. G. N.; Eiras, S. P.; Albuquerque, Y. D. T.; Richter, E. M.; J. Braz. Chem. Soc. 2009, 20, 1249; dos Santos, W. T. P.; de Almeida, E. G. N.; Ferreira, H. E. A.; Gimenes, D. T.; Richter, E. M.; Electroanalysis 2008, 20, 1878; Gimenes, D. T.; dos Santos, W. T. P.; Tormin, T. F.; Munoz, R. A. A.; Richter, E. M.; Electroanalysis 2010, 22, 74.

19. Medeiros, R. A.; Lourenção, B. C.; Rocha-Filho, R. C.; Fatibello-Filho, O.; Anal. Chem. 2010, 82, 8658; Silva, W. C.; Pereira, P. F.; Marra, M. C.; Gimenes, D. T.; Cunha, R. R.; da Silva, R. A. B.; Munoz, R. A. A.; Richter, E. M.; Electroanalysis 2011, 23, 2764.

20. Silva, R. A. B.; Gimenes, D. T.; Tormin, T. F.; Munoz, R. A. A.; Richter, E. M.; Anal. Methods 2011, 3, 2804; Tormin, T. F.; Cunha, R. R.; Richter, E. M.; Munoz, R. A. A.; Talanta 2012, 99, 527.

21. Gimenes, D. T.; dos Santos, W. T. P.; Munoz, R. A. A.; Richter, E. M.; Electrochem. Commun. 2010, 12, 216.

22. Gimenes, D. T.; Pereira, P. F.; Cunha, R. R.; da Silva, R. A. B.; Munoz, R. A. A.; Richter, E. M.; Electroanalysis 2012, 24, 1805.

23. dos Santos, W. T. P.; Ceolin, M. P.; de Albuquerque, Y. D. T.; Richter, E. M.; Quim. Nova 2007, 30, 1754.

24. dos Santos, W. T. P.; Azevedo E. F.; Richter, E. M.; Albuquerque, Y. D. T.; Quim. Nova 2009, 32, 2412.

25. Pedrotti, J. J.; Angnes, L.; Gutz, I. G. R.; Electroanalysis 1996, 8, 673.

26. Gandini, D.; Michaud, P. A.; Duo, I.; Mahe, E.; Haenni, W.; Perret, A.; Comninellis, C.; New Diamond Front. Carbon Technol. 1999, 9, 303.

27. Salazar-Banda, G. R.; de Carvalho, A. E.; Andrade, L. S.; Rocha-Filho, R. C.; Avaca, L. A.; J. Appl. Electrochem. 2010, 40, 1817. 